\title{
CARCINOID HEART DISEASE: NTATION, DIAGNOSIS, AND MANAGEMENT
}

$\mathrm{T}$ ricuspid and pulmonary valve regurgitation usually occurs as a secondary phenomenon caused by dilatation of the valve ring secondary to right ventricular failure or pulmonary hypertension, respectively. Primary diseases of the tricuspid or pulmonary valves are uncommon, but the more likely causes might include congenital abnormalities, rheumatic heart disease, or infective endocarditis. Carcinoid heart disease is a rare, but interesting and important cause of intrinsic tricuspid and pulmonary valve disease leading to significant morbidity and mortality caused by right heart failure. When treated medically, and in appropriate cases surgically, significant benefits in overall quality of life and long term survival can be achieved. We review the current literature regarding the pathophysiological basis of the disease, the cardiovascular complications, and the currently available treatment strategies.

\section{AETIOLOGY AND PATHOPHYSIOLOGY}

Carcinoid tumours are rare neuroendocrine malignancies arising from neural crest amine precursor uptake decarboxylation cells. Approximately $90 \%$ of all carcinoid tumours are located in the gastrointestinal system of which the most common sites are the appendix and terminal ileum. Other less common sites include the bronchus and gonads. The most malignant of the carcinoid tumours tend to arise from the ileum and must be invasive or metastasise to produce the carcinoid syndrome which is characterised by facial flushing, intractable secretory diarrhoea, and bronchoconstriction. The incidence of carcinoid tumours is approximately 1 in 75000 of the population $^{1}$ of whom about $50 \%$ develop carcinoid syndrome. Once the carcinoid syndrome has developed, approximately $50 \%$ of these patients develop carcinoid heart disease which typically causes abnormalities of the right side of the heart.

Usually, only carcinoid tumours that invade the liver result in pathological changes to the heart. The cardiac manifestations are caused by the paraneoplastic effects of vasoactive substances such as 5-hydroxytryptamine (5-HT or serotonin), histamine, tachykinins, and prostaglandins released by the malignant cells rather than any direct metastatic involvement of the heart. Ordinarily, the vasoactive tumour products are inactivated by the liver, lungs, and brain, but the presence of hepatic metastases may allow large quantities of these substances to reach the right side of the heart without being inactivated by the liver.

The characteristic pathological findings are endocardial plaques of fibrous tissue that may involve the tricuspid valve, pulmonary valve, cardiac chambers, venae cavae, pulmonary artery, and coronary sinus. The fibrous reaction may involve not only the valve leaflets, but also the subvalvar apparatus including the tendinous chords and papillary muscles of the tricuspid valve, and more rarely the mitral valve in cases with left sided involvement. The fibrous tissue in the plaques results in distortion of the valves leading to either stenosis, regurgitation, or both. The preferential right heart involvement is most likely related to inactivation of the vasoactive substances by the lungs. In the $5-10 \%$ of cases with left sided valvar pathology, one should suspect either extensive liver metastases, bronchial carcinoid, or a patent foramen ovale. ${ }^{2}{ }^{3}$

Although the precise cause for the plaque formation is not entirely clear, the direct actions of serotonin and bradykinin have been implicated by animal studies. ${ }^{4}$ This finding is corroborated by the observation that the appetite suppressant drug fenfluramine, which releases serotonin, has been noted to cause valvar distortion similar to that seen in carcinoid heart disease. ${ }^{5}$ See end of article for authors'
affiliations

Correspondence to: Dr Rajdeep S Khattar, Manchester Heart Centre, Manchester Royal Infirmary, Oxford Road, Manchester M13 9WL, UK; rskhattar@ hotmail.com

\section{NATURAL HISTORY}

Many carcinoid tumours are slow growing and follow a prolonged course of up to 20 or more years from the development of the carcinoid symptoms. However, the development of cardiac pathology in the carcinoid patient heralds a decline in clinical outcome. Three year mortality data for patients with carcinoid heart disease shows a 31\% survival rate, whereas carcinoid patients without cardiac involvement have approximately twice the survival rate. One study showed mean survival in those with carcinoid heart disease to be reduced to 1.6 years compared to 4.6 years in those without cardiac involvement. ${ }^{6}$ Nevertheless, there is good evidence to suggest that 
treatment of the cardiac aspects of carcinoid not only improves symptoms but increases longevity. ${ }^{7}$

\section{CLINICAL PRESENTATION}

There needs to be a high index of clinical suspicion to diagnose carcinoid heart disease. Carcinoid symptoms typically occur between the fifth and seventh decades of life with a mean age of 55-60 years. ${ }^{8-11}$ The time period between onset of symptoms and diagnosis of carcinoid heart disease usually approximates to $24-28$ months but may be as long as five years. ${ }^{911}$ The mean time lag to surgery, depending on cardiac symptoms and valvar disease severity, is approximately 24 months. ${ }^{9}$ Obvious features of flushing, diarrhoea, and bronchospasm should draw attention to the possibility of the carcinoid syndrome and, as discussed previously, patients with florid carcinoid symptoms have a 50\% chance of cardiac involvement. In such patients, physical examination usually reveals a systolic murmur along the left sternal edge, produced by tricuspid regurgitation; concomitant murmurs of pulmonary stenosis or regurgitation may also be present. Pellagra with dermatitis of sun-exposed areas may also be seen secondary to the high turnover of nicotinic acid metabolised by the tumour.

The vast majority of patients with cardiac involvement present with signs of right heart failure secondary to severe dysfunction of the tricuspid and pulmonary valves. In a large US case series, $97 \%$ of the 74 patients with cardiac involvement had tricuspid valve disease, of whom $90 \%$ displayed moderate or severe tricuspid regurgitation; smaller numbers had coexistent tricuspid stenosis. Pulmonary valve pathology was noted in $88 \%$ of patients, of whom $81 \%$ had pulmonary regurgitation and 53\% had pulmonary stenosis. ${ }^{6}$ In the same series, only $7 \%$ had left sided involvement, in whom the most typical feature was mild to moderate mitral regurgitation; left ventricular systolic function was not usually affected.

Two unusual cases reported carcinoid heart disease presenting as pure right heart failure in the absence of the typical features of carcinoid syndrome. ${ }^{12}$ A further unique case presented with the carcinoid syndrome and right heart failure secondary to constrictive pericarditis rather than tricuspid or pulmonary valve dysfunction. ${ }^{13}$

Cardiac metastases of carcinoid tumours are extremely rare. In the largest series of 11 patients derived from the Mayo clinic, carcinoid syndrome was present in all patients and all metastases were intramyocardial in location. ${ }^{14}$

It is noteworthy that patients with carcinoid heart disease may have labile blood pressure with either pronounced hypotension or hypertension, depending on the relative quantities of the individual vasoactive substances in the circulation. Serotonin, for example, can lead to tachycardia and hypertensive crisis refractory to conventional treatment.

\section{DIAGNOSIS}

Basic screening with a 12 lead ECG and chest $x$ ray is of limited value. Between $30-50 \%$ of ECGs are normal; nonspecific ST segment changes and sinus tachycardia are the most common abnormal findings and p pulmonale or right bundle branch block may also be seen on occasion. ${ }^{6}$ The chest $x$ ray is also largely unhelpful as around $50 \%$ are normal and the remainder have non-specific changes such as increased cardiothoracic ratio, blunting of the costophrenic angles, or small pulmonary nodules. ${ }^{68}$

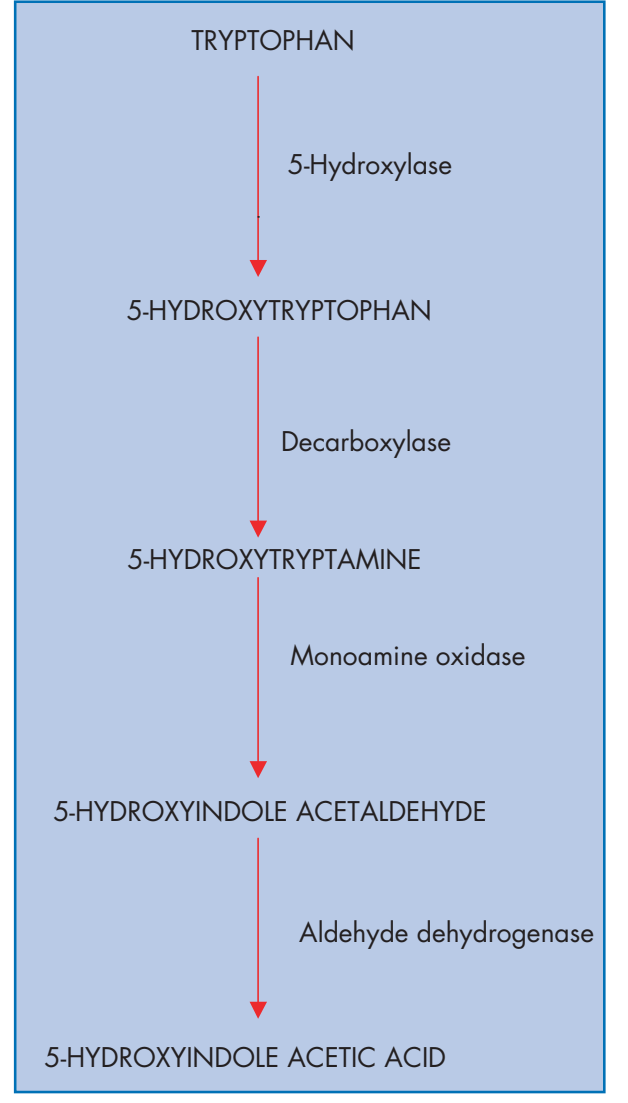

Figure 1 Biochemical pathway for the synthesis and degradation of serotonin (5-hydroxytryptamine).

The two key investigations for the diagnosis of carcinoid heart disease are 24 hour urinary excretion of 5-hydroxyindole acetic acid (5-HIAA) and transthoracic echocardiography.

\section{Biochemical screening}

Metastatic carcinoid tumours take up the amino acid tryptophan and convert it to serotonin (fig 1). The latter is the biologically active molecule of the metabolic pathway and appears to play a role in the pathogenesis of some carcinoid symptoms including diarrhoea and bronchoconstriction. Serotonin is ultimately broken down into 5-HIAA which accounts for more than $95 \%$ of the urinary excretion. Patients with carcinoid syndrome therefore have raised concentrations of 24 hour urinary 5-HIAA. In a large series of patients with carcinoid syndrome and cardiac involvement the mean 24 hour urinary excretion of 5-HIAA was up to 10-fold higher than the reference value (reference value $<50 \mu \mathrm{mol} / \mathrm{l}$ per 24 hours). Some studies suggest that a positive correlation exists between urinary concentrations of 5-HIAA, disease progression, and worsening prognosis. ${ }^{89}$ This has been attributed to the fact that higher circulating concentrations of vasoactive substances produced by the tumour (especially 5-HT causing fibroblast proliferation) are likely to induce more cardiac damage. ${ }^{89}$ In patients with both right and left sided carcinoid heart disease, urinary values of 5-HIAA appear to be higher in patients without interatrial shunts compared to those with interatrial shunts, suggesting greater disease activity in the former group. ${ }^{10}$ It should be noted that diets rich in bananas, nuts, pineapple, kiwi, and avocado can cause modest rises in 
urinary excretion of 5-HIAA and thereby lead to false positive results. Conversely the anti-Parkinson's disease drug levodopa can falsely lower urinary 5-HIAA concentrations.

Notably, carcinoid tumours may also be associated with raised plasma concentrations of gastrin, glucagon, insulin, adrenocorticotropic hormone (ACTH), parathyroid hormone, and calcitonin.

\section{Transthoracic echocardiography}

The main predictor of clinical outcome in patients with carcinoid syndrome is the extent and severity of cardiac involvement. Therefore echocardiography plays a central role in the diagnostic and prognostic evaluation of this condition. In patients with carcinoid heart disease, right atrial and right ventricular enlargement is present in up to $90 \%$ of cases and ventricular septal wall motion abnormalities are seen in almost half of the cases. ${ }^{6}$ The tricuspid valve leaflets and subvalvar structures are often thickened, shortened and retracted, leading to incomplete coaptation and usually moderate or severe tricuspid regurgitation (fig 2). The continuous wave Doppler profile of tricuspid regurgitation shows a characteristic dagger shaped spectrum with an early peak pressure and rapid decline (fig 3 ). The pulmonary valve may also be thickened and retracted, leading to pulmonary regurgitation and less commonly, pulmonary stenosis. The
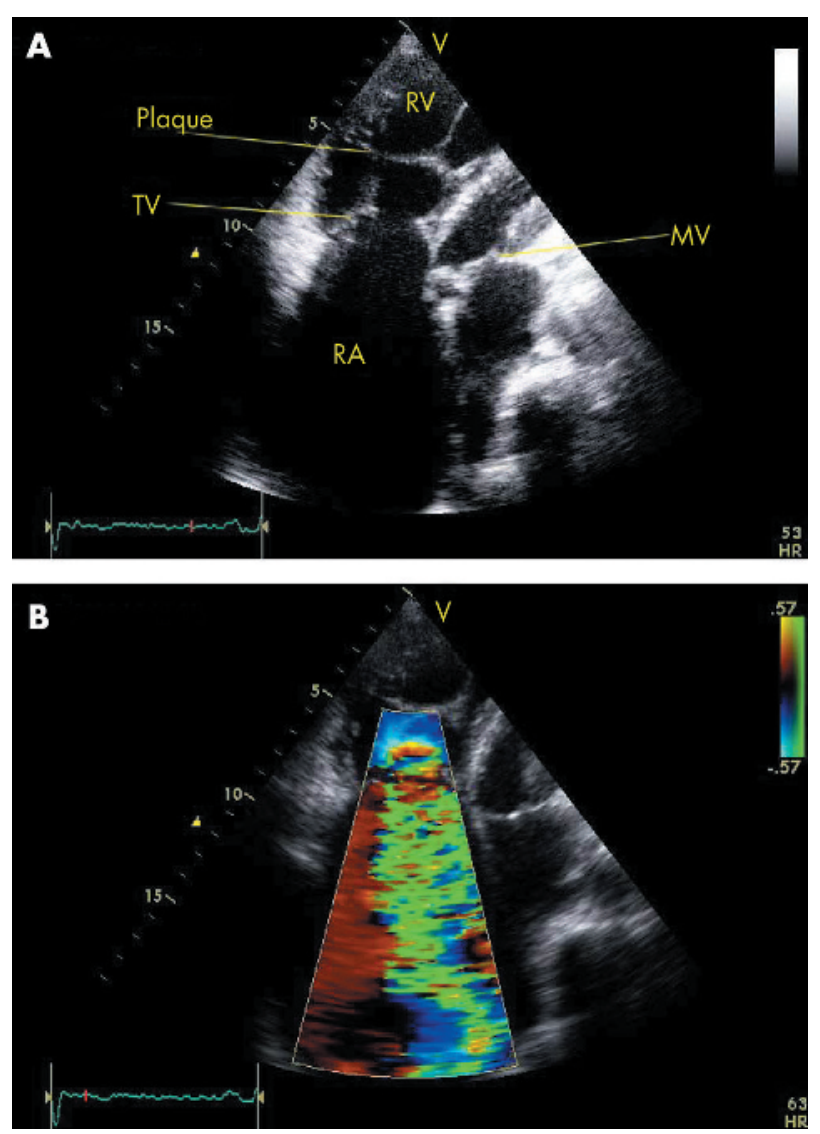

Figure 2 (A) Transthoracic echocardiogram in a modified apical four chamber view showing thickened and rigid tricuspid valve (TV) leaflets in an abnormal, fixed open position during systole. Endocardial plaque formation of the subvalvar apparatus is also notable. (B) Colour flow Doppler imaging in the same view shows severe tricuspid regurgitation through a wide regurgitant orifice. $M V$, mitral valve; RA, right atrium; $\mathrm{RV}$, right ventricle. combination of severe tricuspid regurgitation and pulmonary stenosis is particularly problematic as this further exacerbates the tricuspid regurgitation and worsens right heart failure. Interestingly, calcification of affected valves is rare and may be considered a notable negative echocardiographic feature of carcinoid heart disease.

\section{TREATMENT}

In the early non-metastatic phase, surgical resection of the carcinoid tumour tends to be curative. In those with the carcinoid syndrome, treatment tends to be palliative as metastatic spread has already occurred. Relief of symptoms can be achieved surgically by debulking the tumour, and sometimes, in those with hepatic metastases, by hepatic artery ligation or embolisation.

Patients with cardiac involvement tend to have higher circulating concentrations of 5-HIAA and more advanced disease. The principles of management of patients with carcinoid heart disease can be divided into the treatment of right heart failure, pharmacotherapy to reduce the secretion of tumour products, and surgical/interventional treatment of valvar pathology.

\section{Heart failure management}

General measures for the treatment of heart failure include salt and water restriction, and monitoring of fluid balance and weight. General mobility and compression stockings can help to prevent the development of deep venous thrombosis and epithelial breakdown from gross peripheral oedema. Right heart failure can be successfully treated with a combination of loop diuretics and digoxin. Often, loop diuretics alone are enough to achieve sufficient fluid loss, but if additional diuresis is required, the judicious coadministration of a thiazide diuretic usually produces the desired effect. ${ }^{15}$ Digoxin is believed to help with right ventricular contractility although the data on pure right sided heart failure (without concomitant lung disease or pulmonary hypertension) are limited. ${ }^{7}$

\section{Pharmacotherapy}

The use of somatostatin analogues has been noted to provide symptomatic improvement and improved survival in patients with carcinoid heart disease. Octreotide is an eight amino acid peptide that, by binding to somatostatin receptors, has

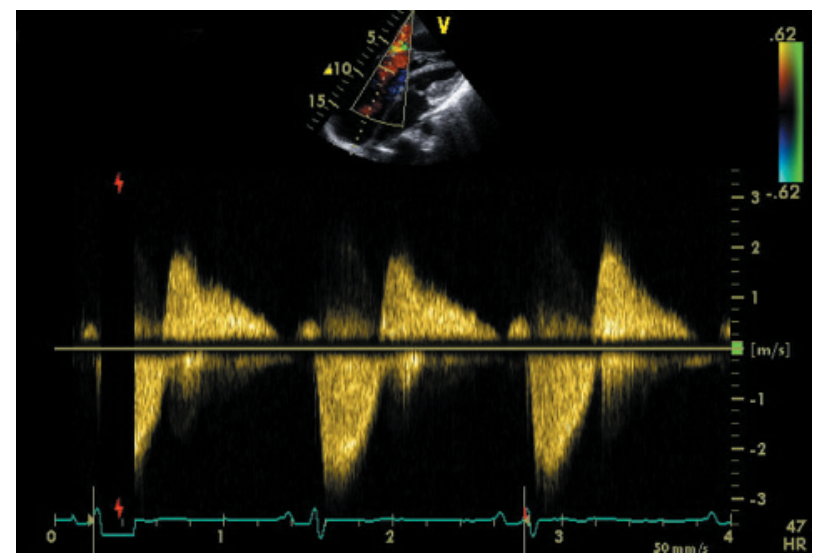

Figure 3 Continuous wave Doppler profile of severe tricuspid regurgitation showing the characteristic dagger shaped spectrum with an early peak pressure and rapid decline. 
the direct effect of reducing the vasoactive peptides that provoke the carcinoid syndrome. Treatment with octreotide gives rise to both directly observable clinical benefit and measurable biochemical improvement. About $70 \%$ of patients obtain symptomatic relief from diarrhoea and flushing, showing a decrease in measurable 5-HIAA urinary secretion and serum 5 -HT concentrations. ${ }^{16}$ Octreotide is usually administered subcutaneously in 2-4 divided doses varying between $50-1500 \mu \mathrm{g} /$ day. ${ }^{17}$ Both blood pressure and blood glucose should be carefully monitored when administering the drug. A newer somatostatin analogue, lanreotide (or BIM23014, angiopeptin and somatuline), may be used as an alternative to octreotide with the advantage of less frequent administration. ${ }^{18}$

There is some evidence that the use of leucocyte interferonalpha controls the secretion of tumour products. It stimulates $\mathrm{T}$ lymphocytes and can produce a notable reduction in tumour size with evidence of survival benefit. Interferonalpha can be used in conjunction with somatostatin antagonists. Unfortunately, however, there are no data to suggest that either interferon or octreotide can cause any regression of the cardiac damage caused by carcinoid disease.

\section{Surgical and interventional treatment}

In selected cases, balloon valvoplasty of the right sided valves has produced symptomatic improvement in patients with stenotic tricuspid or pulmonary valves, although recurrent symptoms have been observed. In suitable candidates, valve surgery is the only definitive treatment for severe right heart failure, as the carcinoid syndrome may produce pronounced morphological defects of the right sided valves. Patients with carcinoid heart disease usually die as a result of severe tricuspid regurgitation ${ }^{7}$ rather than carcinomatosis. Therefore, consideration should be given to the suitability of a patient for valve surgery even in metastatic disease, unless the metastatic process is likely to lead to imminent demise. It is usually considered preferable to operate early or soon after the onset of cardiac symptoms, as delay can result in worsening right ventricular failure and increase the risk of surgery. Perioperative management may involve the use of an intravenous bolus or infusion of octreotide to reduce the risk of intraoperative hypotension. Ketanserin given orally for the week leading up to surgery helps to reduce the risk of a hypertensive crisis, ${ }^{19}{ }^{20}$ but can cause QT prolongation and should therefore be given cautiously to prevent the risk of ventricular arrhythmias. Antihistamines are also used before surgery to prevent flushing and bronchospasm; corticosteroids can be used to reduce bradykinin production.

In the tricuspid position, mechanical valvar prostheses are believed to be adequately durable and relatively unaffected by the vasoactive substances causing the original valve pathology. However, their use is disadvantaged by the fact that carcinoid patients often have extensive liver metastases and therefore are at very high risk from bleeding as a result of the mandatory use of anticoagulant treatment. Bioprosthetic valves might, on balance, be preferable as anticoagulation can be avoided, and the life expectancy of the patient is likely to be shorter than that of the valve. ${ }^{7}$ Unfortunately, tricuspid valve repair is not usually feasible as the leaflets are so restricted that there is a strong possibility of residual postrepair stenosis. There is some debate regarding the optimal surgical management of the pulmonary valve; the two options include valvectomy or valve replacement. Although the data are sparse, a recent small study of 22 patients

\section{Carcinoid heart disease: key points}

- Carcinoid tumours are rare neuroendocrine malignancies mostly arising within the gastrointestinal system, particularly the ileum and appendix

- The carcinoid syndrome, characterised by cutaneous flushing, secretory diarrhoea, and bronchospasm, occurs secondary to the paraneoplastic effects of vasoactive tumour products such as serotonin and only occurs in the presence of metastatic spread

- Raised mean 24 hour urinary excretion of 5-hydroxyindole acetic acid (5-HIAA), an end product of serotonin metabolism, is a key diagnostic finding

- Carcinoid heart disease occurs in approximately $50 \%$ of patients with the carcinoid syndrome and usually heralds a worsening prognosis

- The vast majority of patients with carcinoid heart disease develop right heart failure caused by severe dysfunction of the tricuspid and pulmonary valves

- Palliation of symptoms and prolonged survival can be achieved with appropriate medical treatment and valvar surgery in selected patients with carcinoid heart disease

suggested that pulmonary valve replacement reduced the risk of right heart dilatation postoperatively. ${ }^{21}$

In general, there is evidence to support an increase in both longevity and quality of life in patients successfully treated by surgery. ${ }^{70} 22$ However, those over the age of 60 years are considered to have a high surgical mortality, with one study quoting a $63 \%$ death rate, ${ }^{23}$ possibly even higher in patients with significant co-morbidity. More reassuringly, a recent small study suggests that preoperative control of carcinoid activity with drug treatment, followed by meticulous perioperative anaesthetic and surgical care, may lead to a more acceptable mortality rate of approximately $20 \%$, even in patients with left sided involvement. ${ }^{10}$

\section{ACKNOWLEDGEMENTS}

The authors state that they have read the above article and agree with its content. They also confirm that it has not been published elsewhere, nor is it for consideration in any other journal for publication. There is no conflict of interest from any party.

\section{Authors' affiliations}

D J Fox, R S Khattar, Manchester Heart Centre, Manchester Royal Infirmary, Oxford Road, Manchester, UK

\section{REFERENCES}

1 Bassan MD, Ahlman H, Wangberg B, et al. Biology and management of the midgut carcinoid syndrome. Am J Surg 1993;165:288-97.

2 Schweizer W, Gloor F, Von Bertrab R, et al. Carcinoid heart disease with left sided lesions. Circulation 1964;29:253-7.

3 Strickman NE, Rossi PA, Massumkhani GA, et al. Carcinoid heart disease: a clinical, pathologic and therapeutic update. Curr Prob Cardiol 1982;6:1-42.

4 Spatz M. Pathogenetic studies of experimentally induced heart lesions and their relation to carcinoid syndrome. Laboratory Investigation $1964 ; 13: 288-300$.

5 Khan MA, Herzog CA, St Peter JV, et al. The prevalence of cardiac valvular insufficiency assessed by transthoracic echocardiography in obese patients treated with appetite suppression drugs. N Engl J Med 1998;339:713-18.

- Important study demonstrating the toxic effects of increased circulatory 5-HT concentrations caused by appetite suppression drugs, mimicking carcinoid and causing endocardial fibrosis.

6 Pellikka PA, Tajik AJ, Khandheria BK, et al. Carcinoid heart disease. Clinical and echocardiographic spectrum in 74 patients. Circulation 1993;87: $1188-97$.

Largest series of carcinoid patients with comprehensive echocardiographic data demonstrating the spectrum of structural valvar and cardiac abnormalities, clinical presentations, and therapeutic options. 
7 Connolly HM, Nishimura RA, Smith HC, et al. Outcome of cardiac surgery for carcinoid heart disease. J Am Coll Cardiol 1995;25:410-16.

8 Westberg G, Wangberg H, Ahlman C, et al. Prediction of prognosis by echocardiography in patients with midgut carcinoid syndrome. $\mathrm{Br} /$ Surg 2001:88:865-82.

9 Moller JE, Connolly HM, Rubin J, et al. Factors associated with the progression of carcinoid heart disease. N Engl J Med 2003;348:1005-15.

- Most recent large study, examining in particular key diagnostic features to look for in patients which may herald an adverse or poorer outcome.

10 Connolly HM, Schaff HV, Mullany CJ, et al. Surgical management of left sided carcinoid heart disease. Circulation 2001;104:136-40.

- Case series of 11 patients with left sided carcinoid heart disease showing improved long term outcome in operative survivors. The authors conclude that cardiac surgery should be considered early in appropriate patients

11 Zuetenhorst JM, Bonfrer JMGM, Korse CM, et al. The role of urinary 5hydroxyindoleacetic acid excretion and plasma levels of atrial natriuretic peptide, transforming growth factor beta and fibroblast growth factor. Cancer 2003;97:1609-15.

12 Sirois I, Pothier J, Couture C, et al. Atypical presentation of carcinoid syndrome. Can J Cardiol 1999;15:469-71.

13 Johnson SD, Johnson PW, O'Rourke D. Carcinoid constrictive pericarditis. Heart 1999;82:641-3.

14 Pandya U, Pellikka P, Enriquez-Sarano M, et al. Metastatic carcinoid tumour to the heart: echocardiographic-pathologic study of 11 patients. J Am Coll Cardiol 2002;40:1328.
15 Warrell DA, Cox TM, Firth JD, Benz EJ, eds. Oxford textbook of medicine, 4th ed. Oxford: Oxford University Press, 2003.

16 Matthew HK, Robert JM. Carcinoid tumours. N Engl J Med 1999:340:858-68.

17 Anderson AS, Krauss D, Lang R. Cardiovascular complications of malignant carcinoid disease. Am Heart J 1997;66:693-702.

- General review of carcinoid heart disease including historical background of the condition and in-depth discussion of treatment options. The article is supplemented by clear diagrams and high quality echocardiographic images.

18 Janmohamed S, Bloom SR. Carcinoid tumours. Postgrad Med J 1997;73:207-14.

19 Casthely PA, Jablons M, Griepp RB, et al. Ketanserin in the preoperative and intraoperative management of patients with carcinoid tumour undergoing tricuspid valve replacement. Anesth Analg 1986;65:809-11.

20 Houghton K, Carter JA. Perioperative management of carcinoid syndrome using Ketanserin. Anaesthesia 1986;41:596-9.

21 Connolly HM, Schaff HV, Mullany HJ, et al. Carcinoid heart disease: impact of pulmonary valve replacement on right ventricular function and remodelling. Circulation 2002;106(12 suppl I):151-6

- Observational study showing that pulmonary valve replacement appears to be better than simple pulmonary valvectomy with a reduction in right heart size and improved clinical outcome.

22 Defraigne JO, Jerusalem O, Soyeur D, et al. Successful tricuspid valve replacement and pulmonary valvotomy for carcinoid heart disease. Acta Chiurge Belg 1996;96:170-6.

23 Robiolio PA, Rigolin VH, Harrison JK. Predictors of outcome of tricuspid valve replacement in carcinoid heart disease. Am J Cardiol 1995;75:485-8.

\section{LEARNING ON THE WEB}

Case 7: Recurrent pleural effusions, resistant atrial arrhythmias, and abnormal liver function tests: general medicine or cardiology?

N Melikian, J Anderson*, D Lefroy, Departments of Cardiology and Cardiothoracic Surgery*, Hammersmith Hospital, London, UK

A 65 year old man presented with recurrent pleural effusions. Repeated pleural fluid examination and pleural biopsy were unremarkable. Pericardial calcification was noted on admission and attributed to an uncomplicated episode of pericarditis 30 years previously. His symptoms and signs were not thought not to be associated with the heart. While awaiting an open pleural biopsy the patient was admitted with a further pleural effusion, jaundice, resistant atrial arrhythmias, and dyspnoea. Hepatic investigations including autoantibody screen and transjugular liver biopsy were normal.

The significance of these signs and symptoms, the diagnosis, and the management of these problems are discussed in an interactive case presentation.

To access the interactive case visit BMJ Online Learninghttp://cpd.bmjjournals.com/cgi/hierarchy/cpd_node;CBH (free access; registration required)

Correspondence to: Dr N Melikian; drnarbeh@hotmail.com 